\title{
Online Localization of Radio-Tagged Wildlife with an Autonomous Aerial Robot System
}

\author{
Oliver M. Cliff, Robert Fitch and Salah Sukkarieh \\ Australian Centre for Field Robotics \\ University of Sydney, Australia \\ \{o.cliff,rfitch,salah\}@acfr.usyd.edu.au
}

\author{
Debra L. Saunders and Robert Heinsohn \\ Fenner School of Environment and Society \\ Australian National University, Australia \\ \{debbie.saunders,robert.heinsohn\}@anu.edu.au
}

\begin{abstract}
The application of autonomous robots to efficiently locate small wildlife species has the potential to provide significant ecological insights not previously possible using traditional landbased survey techniques, and a basis for improved conservation policy and management. We present an approach for autonomously localizing radio-tagged wildlife using a small aerial robot. We present a novel two-point phased array antenna system that yields unambiguous bearing measurements and an associated uncertainty measure. Our estimation and informationbased planning algorithms incorporate this bearing uncertainty to choose observation points that improve confidence in the location estimate. These algorithms run online in real time and we report experimental results that show successful autonomous localization of stationary radio tags and live radio-tagged birds.
\end{abstract}

\section{INTRODUCTION}

Environmental monitoring robots can provide valuable information to scientists who study natural phenomena. We are interested in autonomous aerial robots that assist ecologists in studying animal behavior. One important application involves localizing animals in the wild which have been caught, instrumented with a radio tag, and released, and periodically relocalizing the animal over weeks or months for monitoring or recapture. Current research has made great advances in general radio source localization, but the goal of reliably localizing wildlife autonomously and online with a flying robot remains elusive. Our aim in this paper is to take a significant step towards this goal by demonstrating autonomous localization of wild birds with an aerial robotic system.

Studying behavior such as the migratory patterns of various animal species has been of interest for over 250 years [3]. Radio tag localization is used in this context instead of visual imagery due to visual occlusion by foliage. Advances in small-scale radio frequency (RF) emitters over the past five decades now permits their use with small animals, such as birds that weigh as little as $75 \mathrm{~g}$. However, the traditional localization process is largely manual and inhibits large-scale data collection [14]. Typically a human must circle potential target areas, often traveling multiple kilometers, and manually adjust receiver gain. The terrain may be difficult to traverse on foot. There is great opportunity for autonomous flying systems to improve data collection efficiency and thus help to resolve longstanding scientific questions that inform wildlife management policies. It is feasible to consider the problem as one of localizing a static source because migratory animals

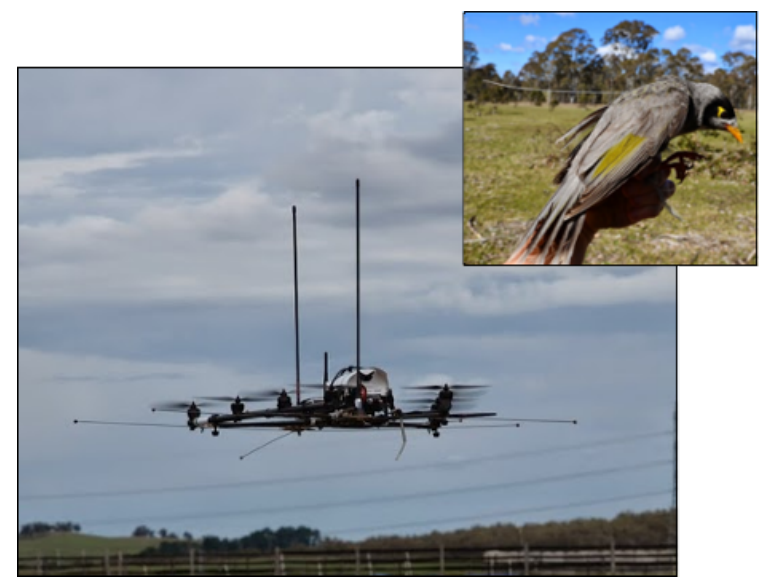

Fig. 1. UAV system in flight. Inset: radio-tagged Manorina Melanocephala.

often remain in an area for weeks, and often remain stationary (within a few meters) over a short time scale (minutes). This assumption is also made in the case of invasive fish [12].

Small aerial robots such as multirotor platforms are suitable for this task because they are easy to deploy and can fly over terrain that is difficult to access on foot, potentially reducing localization time from hours in the manual case to tens of minutes. Further, small aerial robots can operate from sufficient distance to not disturb wildlife. However, it is difficult to design and model a high-performance antenna system that is light enough to be carried. Popular loop aerials [12, 28] are known to be inefficient, especially for low frequency signals. Standard horizontally-mounted directional antennas [16, 22] are affected by unmanned aerial vehicle (UAV) rotors which cause unpredictable irradiance.

We propose an alternative approach based on a two-point phased array: two monopole antennas are mounted to a carrier rail, shown carried by a multirotor platform in Fig. 1. The robot performs a full rotation to produce an unambiguous bearing measurement with a measure of observation uncertainty. Although the time duration of a single observation is roughly $45 \mathrm{~s}$, we found that reasonable localization does not require a large number of observations. We do not consider the detection problem in this work, but instead focus on localization where a signal is present initially. Our contribution is a novel sensor design and algorithms for autonomously locating low-power 
radio tags that have been validated with live birds in the field.

The target location estimate is represented by a gridbased filter, recursively updated following each observation. The measurement bearing (and uncertainty) is obtained by determining the phase shift between an observed gain pattern and the expected Fourier series radiation pattern model through a sliding-correlation technique. We assume that the observation bearing error is normally distributed about a bearing measurement, given some variable uncertainty, and consequently fuse the likelihood of each observation into the target belief. Greedy information-based planning is then used to plan the next observation point online. Estimation in the plane is sufficient in our case because the resulting estimate will generally be used to either confirm the presence of an animal in an area, or to visually locate and catch the animal for sample collection.

We present results from 22 flights and 131 observations, spanning nearly three hours of accumulated flight time. Of these, we performed eight manual flights for system identification and six autonomous flights localizing stationary tags in three different areas. These results validate the performance of the estimation process. Further, we performed three flight trials using live birds where the robot localizes the target while a human tracks its position visually. This evaluation demonstrates the feasibility of localizing birds in the field with low-power RF tags and a small multirotor with limited flight time. Our results reveal the limitations of our approach at the boundary of the stationarity assumption. Finally, we discuss lessons learned and extensions of our approach in practice. We expect our work to lead to further field experiments with other species and comparisons with manual methods.

\section{RELATED WORK}

The general problem of localizing radio-tagged wildlife has recently become a topic of interest in the robotics community. Significant progress has been made in solving the offline estimation problem of localizing an RF emitter. Wagle and Frew [29] propose a Gaussian process-based method and show experimental results from data collected by a fixed-wing UAV. Korner et al. [16] report on offline estimation experiments using a directional antenna and a deterministic range-bearing sensor model. Soriano et al. [26] propose a UAV system for tracking eagles that uses particle filter estimation evaluated using a ground-based testbed. This group has also studied the related problem of localizing a group of static receivers by observing a moving emitter, mounted on an autonomous helicopter [5]. Jensen et al. [13] propose a system for tracking radio-tagged fish evaluated in simulation.

Our focus in this work is online estimation where the robot moves autonomously and the full system is demonstrated experimentally. The problem of designing an online estimator for radio localization and tracking is well studied [19, 18, 20], with emphasis on ground-based systems and an assumed sensor model. Extensive research in online estimation is coupled with optimal sensor placement and planning in [7] and [10]. This paper specifically addresses challenges in employing such a system on a multirotor platform.

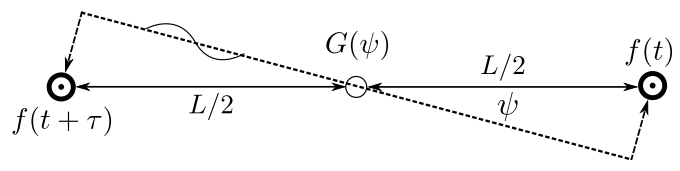

Fig. 2. Two-point phased array. Two monopole antennas are separated by a spacing $L$. This spacing causes a phase offset $\tau$ between the fore and aft antenna as a function of azimuth angle of arrival (AoA) $\psi$. The two signals are summed with a combiner circuit that has an additional (constant) phase offset $\phi$; this then generates a gain pattern $G(\psi) \propto 1+\cos (\tau)$.

Pioneering achievements have been made in autonomous wildlife tracking in recent years. Hook et al. [12] analytically solve the problem of optimally choosing ambiguous bearing measurement locations for localizing a stationary target. Tokekar et al. [28] present a fully autonomous system for detecting and localizing carp; the authors use bearing-only measurements but differ significantly from our approach in implementation by using intersecting conical observations and a polygon belief. Moreover, the authors employ both a different sensor model (loop antenna) and experimental platform (raft). To the best of our knowledge, ours is the first demonstration of autonomous online localization of a live bird with a UAV.

Information-based planning has been studied for over ten years [9]. Recent work has begun to explore sampling-based methods [11]. Numerous applications of information-based planning involve UAVs [10, 15, 21, 24, 25, 27]. In previous work we explored multi-UAV constrained search [8] and UAV planning using formal methods [30, 31]. Here we exploit standard principles in greedily selecting the next observation point from a number of samples.

\section{MULTIROTOR SENSOR MODEL}

Sensor modeling is critical for successful information gathering; the sensor model dictates the quality of observations and therefore directly affects the accuracy of the target estimate. An inaccurate sensor model may result in poor planning decisions that place the robot in states where the target is less likely to be found or tracked. Here we present the hardware and software components of our sensor model and discuss its implementation.

There are three main considerations in designing a sensor model for a multirotor UAV: 1) multipath propagation can cause erroneous observations in cluttered environments, 2) the sensor array must balance weight and quality of readings to maximize the number of useful observations and flight time, and 3) the rotors can cause electromagnetic interfere with a nearby antenna. We approach the first issue by remaining stationary during an observation and obtaining a bearingonly observation. The latter issues are overcome by our novel design of a two-point phased array system.

\section{A. Bearing-only observation}

There are two common techniques for target tracking with radio frequency (RF) beacons: range- and bearing-only observations. Range-only sensor models typically are problematic in cluttered environments as multipath models are undefined with 

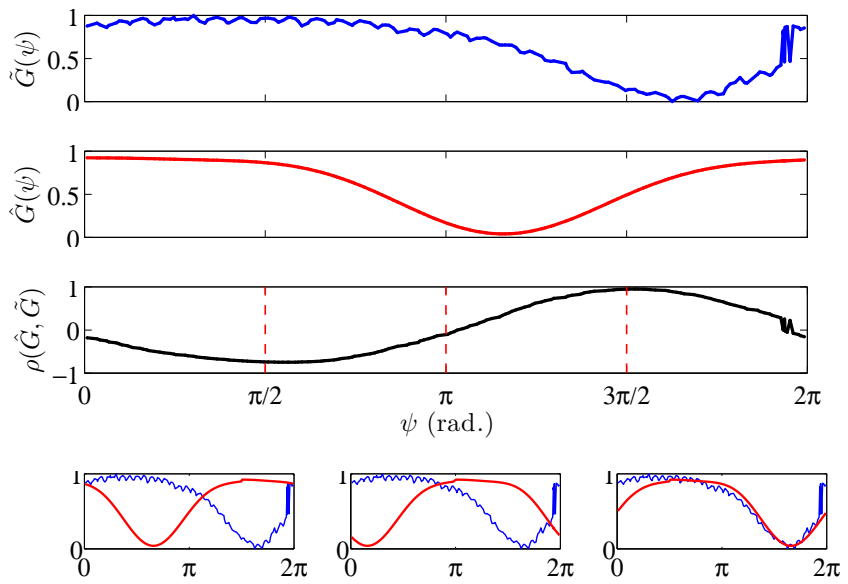

Fig. 3. Illustration of the sliding-correlation waveform matching algorithm. The algorithm obtains a maximum likelihood phase offset by correlating the observed sensor output $\tilde{G}(\psi)$ against the Fourier series model $\hat{G}(\psi+\alpha)$ : $\alpha \in(0,2 \pi]$. The bottom row illustrates $\hat{G}$ shifted by $\alpha=\{-\pi / 2, \pi, 3 \pi / 2\}$.

unknown terrain, e.g., hills and valleys. Hence, we resort to a bearing-only sensor model with a direction finding antenna.

Direction finding antennas are used to passively determine the angle of arrival (AoA) of an emitting source [6]. Most techniques for calculating the AoA for low frequency transmitters are based on the phased array concept and can give an instantaneous reading, e.g., frequency difference of arrival (FDoA) and correlative interferometry (CI) [4]. Commercially available components are not sufficiently sensitive or lightweight for measuring the Doppler shift (in FDoA techniques) or phase shift (for CI) between two sensors in a low-power transmitter $\left(P_{T}<1 \mathrm{~mW}\right)$. For this reason, we mount a directional antenna on the UAV to determine the bearing to the transmitter.

An observation is defined by the UAV system panning one full rotation while the system broadcasts filtered received signal strength indicator (RSSI) readings $\tilde{g}$ at $5 \mathrm{~Hz}$. In the ground-station software, $\tilde{g}$ is associated with an absolute bearing, giving the observed gain pattern $\psi \mapsto \tilde{G}^{k}(\psi)$.

\section{B. Sensor: two-point phased array}

We designed a two-point phased array antenna with a front lobe and back null radiation pattern to account for weight and precision requirements. Shown in Fig. 2, the array consists of two quarterwave monopole antennas situated in front and behind the vehicle center of gravity ( $\mathrm{CoG}$ ) with a spacing $L<\lambda / 4$, where $\lambda$ is the transmitter wavelength. This spacing lags the aft antenna by a phase difference $\tau$ as a function of azimuth AoA $\psi$

$$
\tau=\frac{2 \pi L}{\lambda} \cos (\psi)+\phi .
$$

In Eq. (1), $\phi$ is introduced by an RF combiner with a passive phase offset between the fore and aft antenna. In accordance with (1), if the AoA is perpendicular to the antenna array, the aft antenna phase lag $\tau=\phi$. The interference pattern from this phase difference is then simply $1+\cos (\tau)$. From (1), the asymmetric gain pattern as a function of AoA in $\mathrm{dBi}$ is

$$
G(\psi) \mathrm{dBi}=20 \log _{10}[1+\cos (\tau)]+1 .
$$

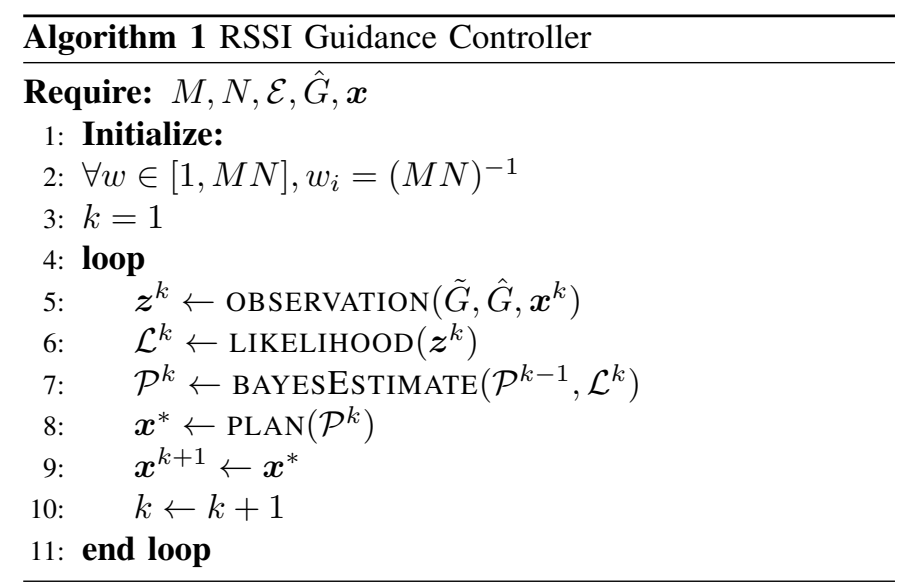

Equation (2) is the ideal case; $\mathrm{dBi}$ is gain relative to a standard half-wave dipole antenna, however with a non-infinite ground plane and inaccuracy in manufacturing and vibrations, the actual pattern is distorted. Further, in Sec. V we introduce an analog circuit to directly sample the RSSI. This causes a distortion of the gain pattern $G(\psi)$. The actual gain pattern $\hat{G}(\psi)$ is empirically evaluated in Sec. VI-A1. The theoretical gain pattern (presented later in Fig. 7(a)p is sampled from Eq. (2) and shows the directionality of the antenna in the foreaft asymmetry.

\section{Bearing-only likelihood function}

The next step in sensor modeling is to derive a bearing-only likelihood function from the gain pattern. To achieve this, we find a phase lag between the observed gain pattern $\tilde{G}^{k}$ and gain pattern model $\hat{G}$ using a sliding-correlation technique with bounded correlation values. We perform a Pearson productmoment correlation $\rho \in[-1,1]$ with a phase lag $\alpha$, s.t.

$$
\rho\left(\hat{G}^{k}, \tilde{G}^{k}\right)[\alpha]=\rho\left(\tilde{G}^{k}(\psi), \hat{G}^{k}(\psi+\alpha)\right) .
$$

where correlation $\rho(X, Y):=\operatorname{cov}(X, Y) /\left(\sigma_{X} \sigma_{Y}\right)$.

From (3), the maximum likelihood estimate for AoA is therefore the phase lag $\alpha$ that yields maximum correlation,

$$
\begin{gathered}
\tilde{\psi}^{k}=\underset{\alpha}{\arg \max }\left\{\rho\left(\tilde{G}^{k}, \hat{G}^{k}\right)[\alpha]\right\}, \\
\rho^{k}:=\rho\left(\tilde{G}^{k}(\psi), \hat{G}^{k}\left(\psi+\tilde{\psi}^{k}\right)\right) .
\end{gathered}
$$

This method is illustrated in Fig. 3

From Eqs. (4) and (5), azimuth AoA estimate $\tilde{\psi}^{k}$ and statistical correlation between expected and observed gain pattern $\rho^{k}$ are associated with the observation state $\boldsymbol{x}^{k}=$ $\left[x^{k}, y^{k}, z^{k}\right]^{T} \in \mathbb{R}^{3}$, i.e., the coordinates of the UAV at observation $k$. This tuple is denoted as measurement $k$, i.e., $\boldsymbol{z}^{k}:=\left(\boldsymbol{x}^{k}, \tilde{\psi}^{k}, \rho^{k}\right)$.

\section{PERCEPTION AND LOCALIZATION}

This section describes the algorithmic components of the system that implement the sensor model (Sec. III) and integrate it into a planning framework. Alg. 1 presents the complete localization method for wildlife tracking: at timestep $k$, the UAV reaches the current observation state $\boldsymbol{x}^{k}$ and performs 


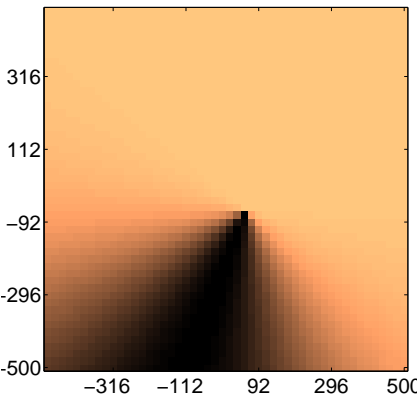

(a) $\rho=0.86, \epsilon=0.65, \hat{\psi}=2.46$

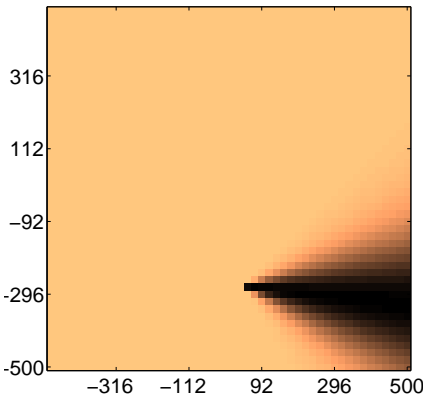

(c) $\rho=0.97, \epsilon=0.31, \hat{\psi}=1.68$

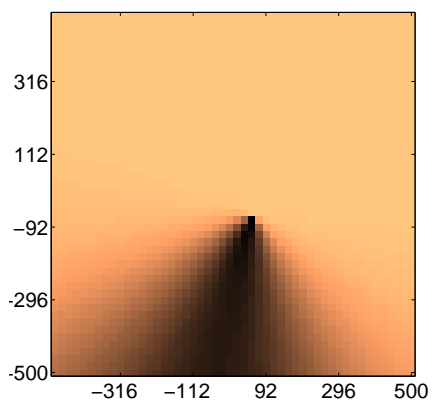

(b) $k=2, H(\boldsymbol{\xi})=9.95$

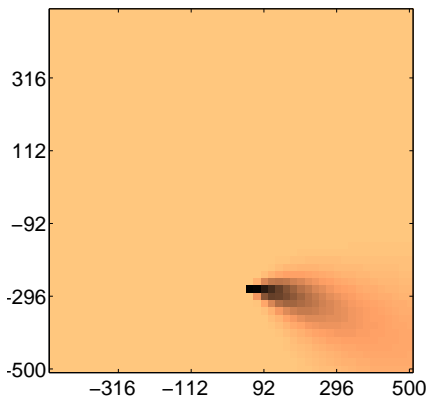

(d) $k=3, H(\boldsymbol{\xi})=8.56$
Fig. 4. Belief representation and data fusion for two consecutive observations. Figs. 4(a) and 4(c) represent the bearing-only likelihood of observation $k=2$ and $k=3$ for this trial; the figures show probability of an observation as a function of correlation $\rho$, bearing error $\epsilon$ and bearing estimate $\tilde{\psi}$. Figs. 4(b) and $4(\mathrm{~d})$ represent the posterior target estimate after these observations, illustrating a reduction in target estimate entropy $H(\xi)$.

a new observation $\boldsymbol{z}^{k}$, calculates the likelihood $\mathcal{L}^{k}$ of $\boldsymbol{z}^{k}$ and fuses this information into the posterior belief $\mathcal{P}^{k}$. The planner then selects the next waypoint $\boldsymbol{x}^{*}$ via the maximum a posteriori (MAP) probability estimate.

\section{A. Belief representation and data fusion}

A grid-based filter is used to maintain the target belief in the plane. Grid-based filters allow resolution-complete optimal recursive estimation if the state space is discrete and consists of a finite number of states [1]. Given that our sensing range is limited, a grid-based approach is preferable to suboptimal methods such as approximate grid-based and particle filters. Further, Kalman filter-based algorithms require the posterior to be Gaussian and hence are not applicable here [1].

Let state $\xi=[\xi, \eta]^{\mathrm{T}} \in \mathcal{E} \subset \mathbb{R}^{2}$ be the target location vector within some predefined state-space $\mathcal{E}$. The target posterior belief at time step (observation) $k$ is a probability mass function and is represented here by an $M \times N$ grid in $\mathbb{R}^{2}$. The grid is composed of cells $\mathcal{P}_{i j}^{k} \equiv \mathcal{P}\left(\boldsymbol{\xi}_{i j} \mid \boldsymbol{z}^{1: k}\right): \forall i \in$ $[1, M], \forall j \in[1, N]$ and $\boldsymbol{z}^{1: k}$ represents the set of sensor measurements $\left\{\boldsymbol{z}^{1}, \boldsymbol{z}^{2}, \ldots, \boldsymbol{z}^{k}\right\}$.

Bayes' theorem is used to obtain a posterior estimate of the target localization $\mathcal{P}^{k}$, given the prior $\mathcal{P}^{k-1}$ and likelihood of observation $k, \mathcal{L}^{k}$, i.e., $\mathcal{P}_{i j}^{k}=\mathcal{L}^{k} \mathcal{P}_{i j}^{k-1} / \eta$ with $\eta$ s.t. $\sum_{i} \sum_{j} \mathcal{P}_{i j}^{k}=1$.

To obtain the likelihood, we assume the probability of measurement $\boldsymbol{z}^{k}$, given the target is at state $\boldsymbol{\xi}_{i j}$, is normally distributed s.t.

$$
\mathcal{L}^{k} \equiv \mathcal{P}\left(\boldsymbol{z}^{k} \mid \boldsymbol{\xi}_{i j}\right)=\frac{1}{\sigma^{k} \sqrt{2 \pi}} \exp \left[-\frac{\left(\gamma_{i j}-\mu^{k}\right)^{2}}{2\left(\sigma^{k}\right)^{2}}\right] .
$$

Here, $\gamma_{i j}$ is the absolute bearing from the observation coordinate to potential target location vector $\boldsymbol{\xi}_{i j}$, i.e.,

$$
\gamma_{i j}=\operatorname{atan} 2\left(\frac{\eta_{i j}-y^{k}}{\xi_{i j}-x^{k}}\right),
$$

and $\mu^{k}=\tilde{\psi}^{k}$ is the AoA estimate. The scale parameter $\sigma^{k}$ corresponds to observation bearing error $\epsilon^{k}$ and is modeled as a piecewise linear function of correlation $\rho$; Sec. VI-A2 presents an empirical evaluation of the function. The estimate for the actual target location is then given by the MAP estimate $\boldsymbol{\xi}^{*}=\arg \max _{\boldsymbol{\xi}_{i j}}\left\{\mathcal{P}_{i j}^{k}\right\}$.

The belief representation and data fusion for two consecutive observations are illustrated in Fig. 4 Note the normally distributed likelihood functions (6) in Figs. 4(a) and 4(c), the target bearing estimate $\tilde{\psi}^{k}$ dictates the orientation of the Gaussian and the correlation quantity $\rho^{k}$ affects the spread of probability about the estimate. The data fusion is illustrated in Figs. 4(b) and 4(d) where the a posteriori target estimate entropy reduces significantly by observation $k=3$.

\section{B. Greedy information gain planner}

Given the sensor model above, we implement greedy information gain planning. The expected information gain of future observations is sampled at static grid locations within a predefined planning radius. The radius limit is important for avoiding environmental hazards such tall power lines.

The quality of a given measurement tuple $z^{k}$ is quantified as the change in Shannon entropy between the prior $\mathcal{P}\left(\boldsymbol{\xi}^{k} \mid \boldsymbol{z}^{1: k}\right)$ and expected posterior $\mathcal{P}\left(\boldsymbol{\xi}^{k+1} \mid \hat{\boldsymbol{z}}^{1: k+1}\right)$ with expected observation $\hat{\boldsymbol{z}}^{k+1}$, i.e.,

$$
I G\left(\hat{\boldsymbol{z}}^{k+1} \mid \boldsymbol{\xi}^{k}\right):=H\left(\boldsymbol{\xi}^{k} \mid \boldsymbol{z}^{1: k}\right)-H\left(\boldsymbol{\xi}^{k+1} \mid \hat{\boldsymbol{z}}^{1: k+1}\right),
$$

where conditional entropy is defined as

$$
H(\boldsymbol{\xi} \mid \boldsymbol{z}):=-\sum_{i}^{M} \sum_{j}^{N} \mathcal{P}\left(\boldsymbol{\xi}_{i j} \mid \boldsymbol{z}\right) \log \mathcal{P}\left(\boldsymbol{\xi}_{i j} \mid \boldsymbol{z}\right) .
$$

Eq. (8) is non-convex and analytically intractable. In order to sample expected information gain at discrete locations, we reformulate Eq. (8) as

$$
\begin{aligned}
& I G\left(\hat{\boldsymbol{z}}^{k+1} \mid \boldsymbol{\xi}^{k}\right)=H\left(\boldsymbol{\xi}^{k} \mid \boldsymbol{z}^{1: k}\right) \\
& \quad-H\left(\boldsymbol{\xi}^{k+1} \mid \hat{\boldsymbol{x}}^{k+1}, \hat{\psi}^{k+1}=\psi^{*}, \hat{\rho}^{k+1}=\rho^{*}\right) .
\end{aligned}
$$

Here, $\psi^{*}$ denotes that the expected bearing measurement $\hat{\psi}^{k+1}$ is given by

$$
\psi^{*}=\operatorname{atan} 2\left(\frac{\eta^{*}-\hat{y}^{k+1}}{\xi^{*}-\hat{x}^{k+1}}\right),
$$

where $\boldsymbol{\xi}^{*}=\sum_{i} \sum_{j} \boldsymbol{\xi}_{i j} \mathcal{P}_{i j}$ and is the expectation of the target location given the prior belief. Note that the correlation 


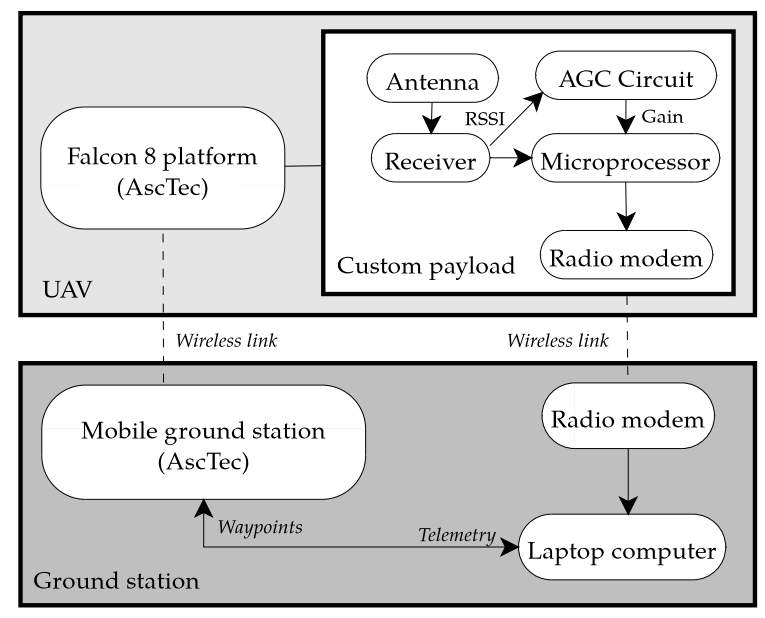

Fig. 5. Diagram of the experimental system.

parameter $\rho^{k}$ scales $I G$ and since we take the maximum, $\rho^{*}$ is set arbitrarily. The next UAV waypoint $\boldsymbol{x}^{*}$ is selected from Eq. (10) s.t.

$$
\boldsymbol{x}^{*}=\underset{\hat{\boldsymbol{x}}^{k+1}}{\arg \max }\left\{I G\left(\hat{\boldsymbol{z}}^{k+1} \mid \boldsymbol{\xi}^{k}\right)\right\} .
$$

This objective function ignores travel cost in order to maximise observation quality. It may be possible to perform more observations per flight by considering travel cost, but we leave this analysis for future work.

\section{EXPERIMENTAL SYSTEM}

Our experimental system comprises a commercial UAV platform, a custom antenna array and sensor payload. Algorithmic components from Sec. IV] are implemented in ROS [23] and executed on a ground-based laptop computer. This section describes the UAV platform and sensor payload components. An overview is shown in Fig. 5 .

The UAV used in our system is the Falcon 8, a commercial eight-rotor platform manufactured by Ascending Technologies with proprietary high-quality flight control and autonomous GPS waypoint-following systems. It is structured around two colinear sets of four rotors with a maximum take-off weight of $2200 \mathrm{~g}$ and payload capacity of $750 \mathrm{~g}$. The platform is connected by wireless communication to a ground station, which can relay telemetry data and accept control commands via USB.

The sensor array is fed into a custom transceiver subsystem that consists of a Radiometrix LMR1 receiver, an ARM 32-bit Cortex-M3 microprocessor mounted to a custom miniaturized printed circuit board, an analog filtering circuit, and a Digi XTend radio modem. These components were chosen such that the total mass of the sensor payload does not exceed the payload capacity of the Falcon 8 . The complete system is shown earlier in Fig. 1

The radio tags regularly transmit an unmodulated on-offkeyed signal with a pulse width of $10 \mathrm{~ms}$ and period of $1.05 \mathrm{~s}$; the receiver RSSI output is an equivalent waveform with an amplitude corresponding to the signal strength at the RF input

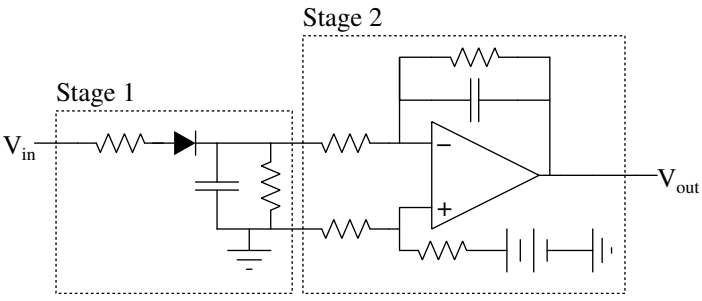

(a) Diagram of the automatic gain control (AGC) circuit

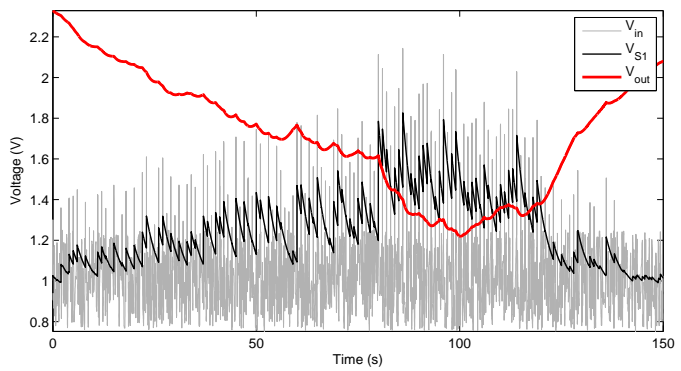

(b) Simulation results for the AGC circuit

Fig. 6. The AGC circuit. The diagram in 6(a) illustrates both stages of the circuit: catch and hold (Stage 1), and integrating amplifier (Stage 2). The plots in Fig. 6(b) show a simulated input pulse of $10 \mathrm{~ms}$ with a period of $1.05 \mathrm{~s}\left(V_{\text {in }}\right)$, the output from Stage $1\left(V_{\mathrm{S} 1}\right)$, and output from Stage $2\left(V_{\text {out }}\right)$.

channel (see $V_{\text {in }}$ in Fig. 6(b)). To avoid using high-powered signal processing components while at the same time receiving high-fidelity RSSI measurements, we designed a simple analog circuit to handle signal processing. The circuit implemented is a typical AGC circuit as seen in Fig. 6(a). Stage 1 is a peak-hold rectifier circuit with a low leakage rate to hold the amplitude of each pulse ( $V_{\mathrm{S} 1}$ in Fig. 6(b)); and Stage 2 acts as an inverting amplifier and integrator circuit to smooth the peak-hold signal and upsample the voltage to the analog-todigital converter (ADC) input range of $0-3.3 \mathrm{VDC}\left(V_{\text {out }}\right.$ in Fig. 6(b) . The filter output $V_{\text {out }}$ is sampled at $5 \mathrm{~Hz}$ by an ADC within the microprocessor subsystem and transmits this packet to the ground station through a radio modem.

\section{EXPERIMENTAL RESULTS}

This section presents experimental validation of our system. First, we present system identification obtained from manual flights with a known tag location in free space. We then provide results from two experiments with autonomous flight: 1) algorithm validation and 2) live bird trials. The aim of the algorithm validation is to localize a stationary tag mounted in the canopy of a tree. Live bird trials were performed with radio-tagged Manorina Melanocephala, a small territorial bird species. In these experiments, observations are taken at $z=50 \mathrm{~m}$ altitude from the launch elevation and take approximately 45 s to complete. Rotation rate for an observation was hand-tuned using data from five preliminary flights. For all autonomous flights, observation positions were computed online using the planner from Sec. IV-B. We use three separate trial sites (Sites A, B and C) known to be within the territory of a target bird. 


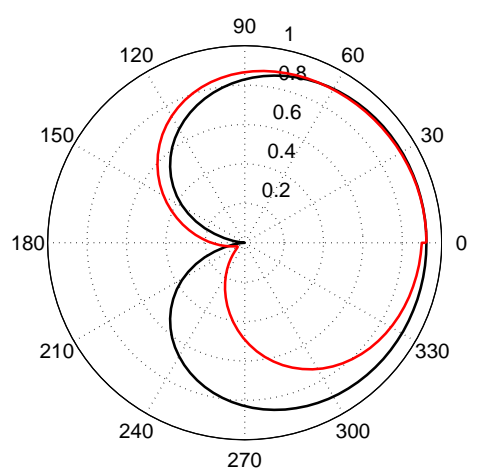

(a) Theoretical gain

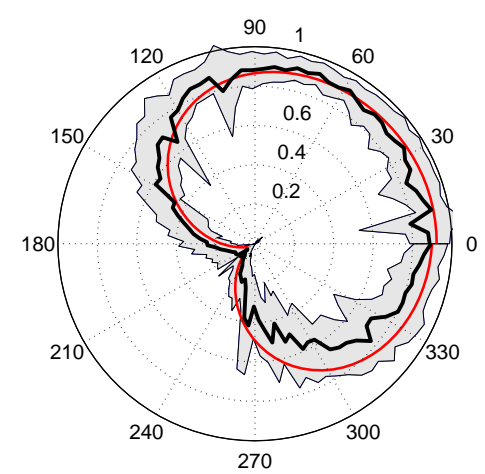

(b) Stationary tag observations

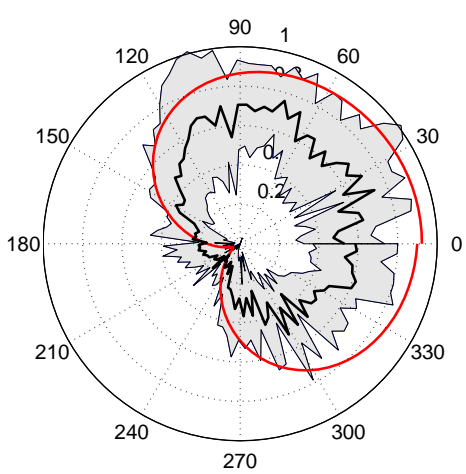

(c) Real bird observations

Fig. 7. Third-order fast Fourier transform gain pattern model $\hat{G}(\psi)$ (red) plotted against: 7(a) the theoretical two-point phased array model $G(\psi)$ (without on-board filter distortion), $7(\mathrm{~b})$ the observations relative to a static tag in the canopy $\tilde{G}_{\text {stationary }}(\psi)$, and $7(\mathrm{c})$ the observations relative to a real bird, moving during observations $\tilde{G}_{\text {bird }}(\psi)$. In $7(\mathrm{~b})$ and 7(c) the mean (solid black line) and standard deviation (shaded gray) normalized gain pattern are shown.
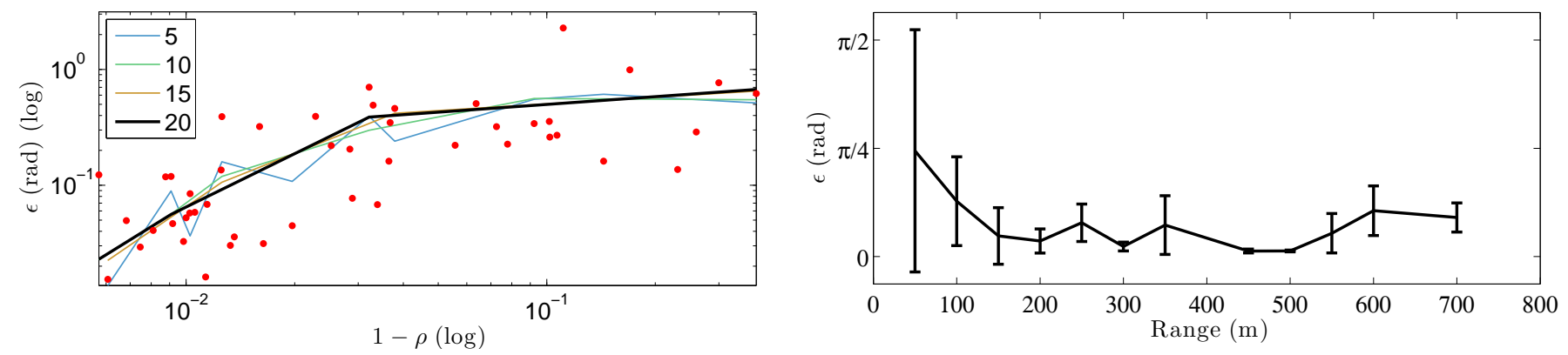

Fig. 8. Plots for the bearing error as a function of $[8(\mathrm{a})]$ gain pattern correlation and 8 (b) range for a stationary transmitter. Bearing error is the deviation from the maximum likelihood bearing to the actual bearing of a known stationary tag. The black piecewise function in Fig. 8(a) was used in determining the variance of our sensor model as a function of gain pattern correlation. Each piecewise function illustrated was built using data segments of size 5, 10, 15 or 20. Note that for clarity, Fig. 8(a) plots the data x-values as $1-\rho$ and is scaled logarithmically as most observations were greater than $\rho=0.9$.

\section{A. System identification}

Eight flights were performed to empirically evaluate: 1) the sensor array gain pattern, 2) bearing error as a function of waveform correlation and 3) observation quality as a function of range to tag. These tests are described below.

1) Empirical gain pattern: In Sec. III-B we derived Eq. (2) for the two-point phased array gain pattern. Due to the onboard filter described in Sec. $\mathrm{V}$, this gain pattern has an attack and decay rate as a function of rotational velocity, distorting the signal (see Fig. 7(a) . To account for this, we use an empirical dataset to compute a discrete Fourier transform offline for the gain pattern as a function of azimuth AoA. We calculated a third-order $(J=3)$ Fourier series from the data using a fast Fourier transform (FFT), i.e. a function

$$
\hat{G}(\psi)=a_{0}+\sum_{j=1}^{J} a_{j} \cos (j w \psi)+\sum_{j=1}^{J} b_{j} \sin (j w \psi) .
$$

Here, $\mathcal{F}=\left\{a_{0: J}, b_{1: J}, w\right\}$ are the Fourier series coefficients.

Figure 7 illustrates the gain pattern from observations at Site A for stationary tags and live birds. For Figs. 7(b) and 7(c), the filtered output from the analog circuit is sampled at $5 \mathrm{~Hz}$ to obtain an observed signal strength $\psi_{\mathrm{tag}} \mapsto \tilde{G}\left(\psi_{\mathrm{tag}}\right): \psi_{\mathrm{tag}} \in$
$(0,2 \pi]$. Note that the live bird gain pattern is noisier with a higher observation variance and does not match the model as well as the stationary tag, causing lower correlations and a more difficult localization problem.

2) Observation bearing error: To determine the likelihood for a given measurement $\boldsymbol{z}^{k}$, we must map the measurement correlation to a bearing error $L(\rho): \rho^{k} \rightarrow \epsilon^{k}$. To achieve this, we assume that observation precision (inversely proportional to variance) is a monotonically increasing function of correlation and accordingly calculate observation variance as a continuous piecewise linear function of correlation. If we take the standard assumption of a (zero mean) standard error distribution for bearing observations, we can validate monotonicity qualitatively by plotting observation bearing error to a known target location as a function of waveform correlation (Fig. 8(a)). The data are divided into partitions of 20 data points and linear regression is performed to produce a continuous piecewiselinear function. We opt for this simple regression model due to the spread and number of data points; optimizing the partitioning would likely yield only marginal improvements. Twenty data points were chosen to maintain monotonicity in precision; Fig. 8(a) illustrates other partitionings. 
TABLE I

LOCALIZATION METRICS FOR A STATIONARY TAG. EACH TAG WAS PLACED IN THE CANOPY OF A SITE WITHIN A TARGET BIRD'S TERRITORY.

\begin{tabular}{|c|c|c|c|c|c|c|c|c|c|c|}
\hline \multirow{2}{*}{ Site } & \multirow{2}{*}{ Trials } & \multirow{2}{*}{$\begin{array}{c}\text { Total } \\
\text { Observations }\end{array}$} & \multirow{2}{*}{$\begin{array}{l}\text { Mean } \\
\text { Error* } \\
(\mathrm{m})\end{array}$} & \multirow{2}{*}{$\begin{array}{c}\text { Mean } \\
\text { Entropy* } \\
\text { (bits) }\end{array}$} & \multicolumn{2}{|c|}{ Mean Distance (m) } & \multicolumn{2}{|c|}{ Correlation $\rho^{\dagger}$} & \multicolumn{2}{|c|}{ Observation Error $\epsilon$ (rad. $)^{\dagger}$} \\
\hline & & & & & $\mu$ & $(\sigma)$ & $\mu$ & $(\sigma)$ & $\mu$ & $(\sigma)$ \\
\hline A & 2 & 11 & 16.43 & 10.56 & 73.3 & $(22.0)$ & 0.951 & $(0.0489)$ & 0.1173 & $(0.0703)$ \\
\hline B & 2 & 11 & 25.2 & 15.7 & 203 & $(95.4)$ & 0.941 & $(0.0355)$ & 0.0691 & $(0.0537)$ \\
\hline \multirow[t]{2}{*}{$\mathrm{C}$} & 2 & 8 & 29.9 & 15.86 & 230 & (118.9) & 0.848 & $(0.175)$ & 0.215 & $(0.349)$ \\
\hline & 6 & 30 & 23.8 & 14.04 & 168.8 & $(78.77)$ & 0.913 & $(0.0865)$ & 0.1338 & $(0.1577)$ \\
\hline
\end{tabular}

$\dagger$ Each observation is independent of parent trial

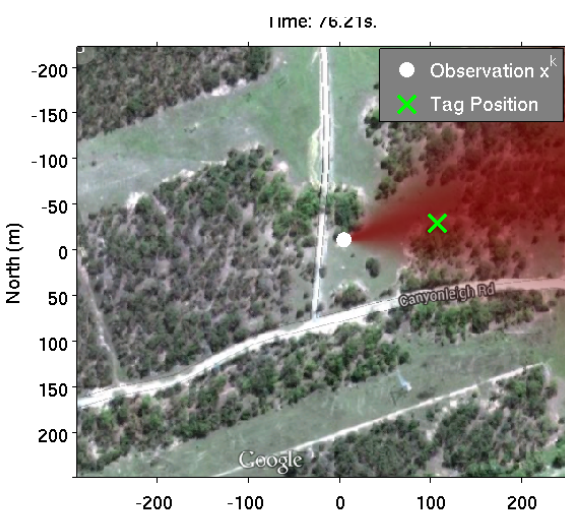

(a) $k=1$

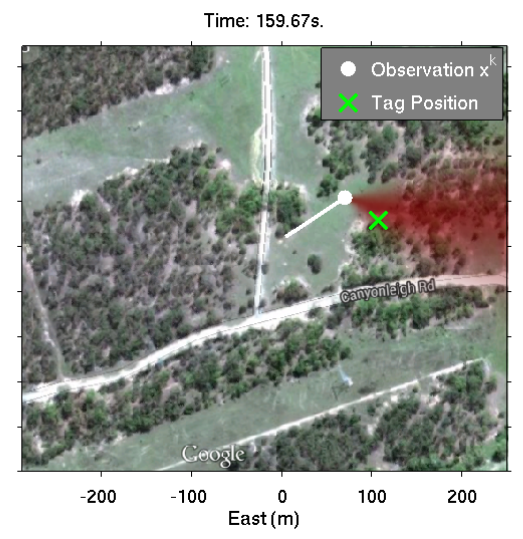

(b) $k=2$

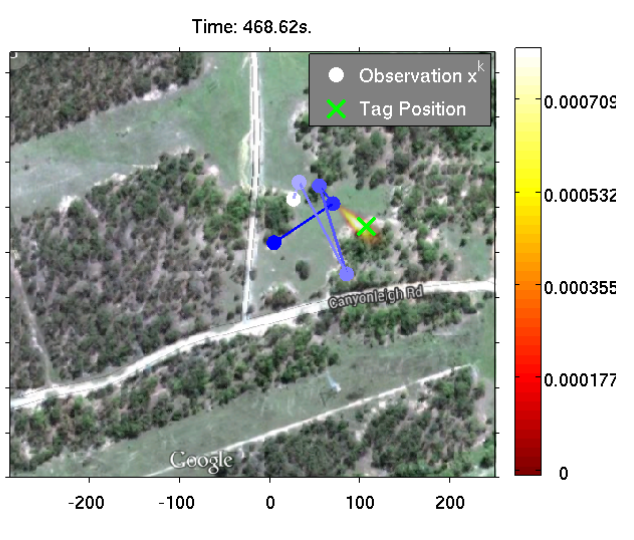

(c) $k=6$

Fig. 9. Localization of a static radio tag in a tree canopy. Figures 9(a) 9(b) and 9(c) illustrate the convergence of the a posteriori belief $\mathcal{P}\left(\boldsymbol{\xi}^{k} \mid \boldsymbol{z}^{1: k}\right)$ of the tag location after the first, second and last observation for this trial, respectively. The belief is represented as a grid with $1 \mathrm{~m}$ resolution.

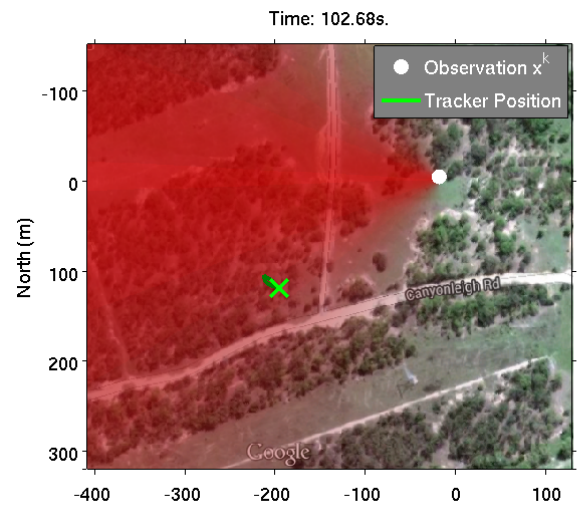

(a) $k=1$

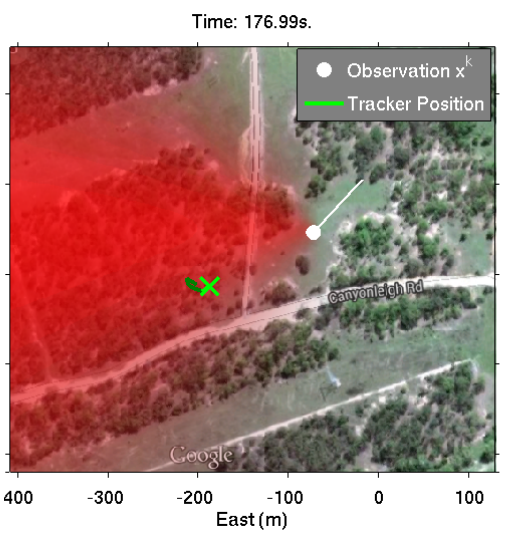

(b) $k=2$

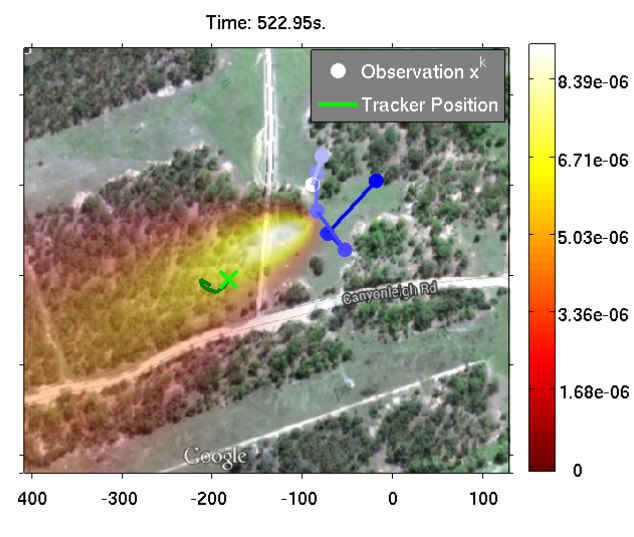

(c) $k=7$

Fig. 10. Localization of the Manorina Melanocephala avian species tagged with a low-power radio transmitter. Figures 10(a) 10(b) and 10(c) illustrate the convergence of the a posteriori belief of the bird location after the first, second and last observation for this trial, respectively. As an indication of the actual bird position, the trajectory of the trackers during an observation (solid light green), and past trajectory (solid dark green) are shown.

3) System range: Figure $8(\mathrm{~b})$ indicates the ideal range of the system as a function of bearing error. To determine the approximate range of the system, we took various observations at iteratively increasing distances from the tag at $50 \mathrm{~m}$ altitude over a flat and uncluttered environment. Figure $8(\mathrm{~b})$ graphs this range as a function of observation bearing error and qualitatively suggests the ideal system range is $100-500 \mathrm{~m}$. Observations closer than $100 \mathrm{~m}$ have a high incidence elevation and thus azimuth gain is not as pronounced; observations beyond $500 \mathrm{~m}$ have a signal strength below the receiver's sensitivity. Closer observations could be taken at a lower altitude however considering time constraints per trial, uncertainty about target location and canopy height, it is justified to keep a consistent height. Additionally, a higher altitude should increase the received signal strength according to the two-ray model, yet this was not observed in practice due to the inherent stochasticity in any low altitude RF application. 


\section{B. Algorithm validation: stationary tag}

Six flights were performed to evaluate localization performance on a stationary tag in a $1000 \times 1000 \mathrm{~m}$ grid with $1 \mathrm{~m}$-resolution cell edges. Table I presents the mean number of observations per trial, mean estimation error and mean entropy of the a posteriori belief of each trial, mean and variance of each observation range to tag, mean and variance of observation bearing error, and the mean and variance of the maximum likelihood correlation of each observation. The former metrics report on the final observation $\left(k=k_{\max }\right)$ of each trial; the latter are calculated over all observations at each site (e.g., 11 observations at Site A). Lower values for all statistical metrics imply higher accuracy, precision and certainty of the tag's position. The observation range is dependent only on environmental factors.

Site A yields better results across all metrics, even though many observations are closer than the ideal system range, most likely suggesting the actual system range is shorter than the free-space case presented in Sec. VI-A Further, the site's landscape was relatively flat with sparser vegetation than other sites and thus yields less dominant multipath propagation. In Sites B and C the algorithm performs sufficiently and consistently localizes the stationary tag to within $30 \mathrm{~m}$.

A typical trial for the stationary tag at Site A is depicted in Fig. 9 showing the a posteriori belief, vehicle trajectory and tag location for observations 1, 2 and 6. Ideally, the planner would be able to circle around the centroid, however due to safety precautions the planner considers waypoints within a $90 \mathrm{~m}$ radius of the UAV home position (the map origin). To account for the aforementioned ideal range, the planning set excludes waypoints within $60 \mathrm{~m}$ of the a priori belief centroid.

\section{Field experiments: radio-tagged avian species}

Following the algorithm validation, we performed three flights from different launch sites to localize small, live birds. Each bird was fitted with a radio transmitter equivalent to the stationary case above. The unique frequency of each radio tag was pre-programmed as a channel in the LMR1 receiver. Prior to each trial, a ground-based human tracker manually located the bird and then recorded its position during the trial.

We present an illustration of a trial at Site A in Fig. 10. Figure 10(a) shows the launch position at approximately $300 \mathrm{~m}$ from the bird in a clearing to allow direct line of sight (LoS) to the UAV system when flying toward the bird. Power lines in the area restrict the planning distance to $90 \mathrm{~m}$ from the home (launch) position, indicated by the $90 \mathrm{~m}$ radius arc in Fig.10(c), Finally, Fig. 10 shows the belief converging to a MAP estimate within $50 \mathrm{~m}$ of the bird - the entropy and accuracy of this trial should improve if the UAV could plan a larger radius around the bird (see stationary tag results in Tab. II).

Results for the second and third trials are similar. These results are less exhaustive and quantitative than those with stationary tags due to two reasons: 1) the inaccuracy of the manual tracking and unpredictable movement of the bird, i.e., if a bird moves between the fourth and fifth observation the manual tracks will be lagged and 2) if the bird moves during an observation, the observation gives the incorrect gain pattern. Further, the planning must take into account the environment (e.g., power lines) and allow an unobstructed LoS to the UAV system for safety precautions, causing difficulty in observing the tag from optimal bearings.

\section{LESSONS LEARNED}

Field testing revealed several interesting lessons regarding antenna performance, transmitter attenuation with live animals, and practical operation. We report these points in this section.

Our two-point phased array antenna significantly outperforms standard $\mathrm{H}$-shaped antennas typically used in UAV applications. Although a full rotation is needed, the resulting high-quality bearing estimate allows for better localisation accuracy than has been achieved with directional antennas offline in similar applications [16]. We also found that our system achieved a detection range that is similar to hand-held systems due to the elevation of the UAV.

We expected significant signal attenuation when moving from the static tag to the live bird case. However, we found such attenuation to be reasonable (Fig. 77). We expect that our system would perform well with other species of similar size. Nocturnal animals which are relatively stationary during the day are good candidates.

One limiting practical factor is short UAV flight time. We operated flights in quick succession by swapping batteries, but longer duration flight would benefit localisation by allowing more observations per flight. Advances in platforms and battery energy density should mediate this issue in future.

\section{DISCUSSION AND FUTURE WORK}

We presented a full UAV system for localizing radio-tagged wildlife and demonstrated autonomous flight. Estimation is based on a Fourier series model of expected RSSI observations trained from data collected in a representative outdoor environment and experimentally validated on Manorina Melanocephala. Our future aim is to continue evaluating this system in field trials with different species. In our experiments so far, we assumed that the radio tag is initially observable. In future work it is important to consider the case where no tag is initially observable, which introduces a search and detect component to the problem, and the issue of when the operator should move [2], in addition to localization. It is also important to explore properties such as submodularity [17] of the objective function (Eq. (12) and algorithms that exploit these properties to maximise information while considering travel cost.

\section{ACKNOWLEDGEMENTS}

This work was supported in part by the Australian Research Council's Linkage Projects funding scheme (project number LP120100448), the Australian Centre for Field Robotics and the New South Wales State Government. Special thanks to Jeremy Randle for expert assistance with flight trials. 


\section{REFERENCES}

[1] M. S. Arulampalam, S. Maskell, and N. Gordon. A tutorial on particle filters for online nonlinear/non-Gaussian Bayesian tracking. IEEE Trans. Signal Process., 50(2): 174-188, 2002.

[2] G. Best, W. Martens, and R. Fitch. A spatiotemporal optimal stopping problem for mission monitoring with stationary viewpoints In Proc. of RSS, 2015.

[3] E. S. Bridge, K. Thorup, M. S. Bowlin, P. B. Chilson, R. H. Diehl, R. W. Fléron, P. Hartl, K. Roland, J. F. Kelly, W. D. Robinson, and M. Wikelski. Technology on the move: recent and forthcoming innovations for tracking migratory birds BioSci., 61(9):689-698, 2011.

[4] G. Brooker. Introduction to sensors for ranging and imaging SciTech Publishing, Inc., 2009.

[5] F. Caballero, L. Merino, I. Maza, and A. Ollero. A particle filtering method for wireless sensor network localization with an aerial robot beacon In Proc. of IEEE ICRA, pages 596-601, 2008.

[6] Y. T. Chan, B. J. Lee, R. Inkol, and Q. Yuan. Direction finding with a four-element Adcock-Butler matrix antenna array. IEEE Trans. Aerosp. Electron. Syst., 37 (4):1155-1162, 2001.

[7] E. W. Frew. Observer trajectory generation for targetmotion estimation using monocular vision. Phd thesis, Stanford University, 2003.

[8] S. K. Gan, R. Fitch, and S. Sukkarieh. Online decentralized information gathering with spatial-temporal constraints Auton. Robot., 37(1):1-25, 2014.

[9] B. Grocholsky. Information-theoretic control of multiple sensor platforms. $\mathrm{PhD}$ thesis, The University of Sydney, 2002.

[10] G. M. Hoffmann and C. J. Tomlin. Mobile sensor network control using mutual information methods and particle filters In Proc. of IEEE AC, pages 32-47, 2010.

[11] G. Hollinger and G. Sukhatme. Sampling-based robotic information gathering algorithms. Int. J. Rob. Res., 33 (9):1271-1287, 2014.

[12] J. Vander Hook, P. Tokekar, and V. Isler. Cautious greedy strategy for bearing-only active localization: analysis and field experiments J. Field Robot., 31(2):296-318, 2014.

[13] A. M. Jensen, D. K. Geller, and Y. Q. Chen. Monte Carlo simulation analysis of tagged fish radio tracking performance by swarming unmanned aerial vehicles in fractional order potential fields J. Intell. Robot. Syst., 74 (1-2):287-307, 2014.

[14] R. E. Kenward. A manual for wildlife radio tagging Academic Press, London, 2001.

[15] A. T. Klesh, P. T. Kabamba, and A. R. Girard. Path planning for cooperative time-optimal information collection. In Proc. of IEEE AC, pages 1991-1996, 2008.

[16] F. Korner, R. Speck, A. H. Goktogan, and S. Sukkarieh.
Autonomous airborne wildlife tracking using radio signal strength In Proc. of IEEE/RSJ IROS, pages 107-112, 2010.

[17] A. Krause and C. Guestrin. Submodularity and its applications in optimized information gathering. $A C M$ Trans. Intell. Syst. Technol., 2(4):32:1-32:20, 2011.

[18] R. X. Li and V. P. Jilkov. Survey of maneuvering target tracking. Part III: Measurement models In Proc. of SPIE SDPST, pages 423-446, 2001.

[19] R. X. Li and V. P. Jilkov. Survey of maneuvering target tracking. Part I: Dynamic models. IEEE Trans. Aerosp. Electron. Syst., 39(4):1333-1364, 2003.

[20] R. X. Li and V. P. Jilkov. Survey of maneuvering target tracking. Part V: Multiple-model methods IEEE Trans. Aerosp. Electron. Syst., 41(4):1255-1321, 2005.

[21] J. Nguyen, N. Lawrance, R. Fitch, and S. Sukkarieh. Energy-constrained motion planning for information \begin{tabular}{|l|l|}
\hline gathering with autonomous aerial soaring & In Proc. of
\end{tabular} IEEE ICRA, pages 3825-3831, 2013.

[22] A. Posch and S. Sukkarieh. UAV based search for a radio tagged animal using particle filters In Proc. of ARAA ACRA, pages 107-112, 2009.

[23] M. Quigley, K. Conley, B. P. Gerkey, J. Faust, T. Foote, J. Leibs, R. Wheeler, and A. Y. Ng. ROS: an opensource robot operating system In Proc. of IEEE ICRA, Workshop on Open Source Software, 2009.

[24] A. Ryan and J. K. Hedrick. Particle filter based information-theoretic active sensing Robot. Auton. Syst., 58(5):574-584, 2010.

[25] P. Skoglar, J. Nygards, and M. Ulvklo. Concurrent path and sensor planning for a UAV - towards an information based approach incorporating models of environment and sensor. In Proc. of IEEE/RSJ IROS, pages 2436-2442, 2006.

[26] P. Soriano, F. Caballero, and A. Ollero. RF-based particle filter localization for wildlife tracking by using an UAV In Proc. of IEEE ISR, pages 239-244, 2009.

[27] J. Tisdale, Z. Kim, and J. Hedrick. Autonomous UAV path planning and estimation. IEEE Robot. Autom. Mag., 16(2):35-42, 2009.

[28] P. Tokekar, D. Bhadauria, A. Studenski, and V. Isler. A robotic system for monitoring carp in Minnesota lakes J. Field Robot., 27(6):779-789, 2010.

[29] N. Wagle and E. Frew. Spatio-temporal characterization of airborne radio frequency environments In Proc. of IEEE GLOBECOM Workshops, pages 1269-1273, 2011.

[30] C. Yoo, R. Fitch, and S. Sukkarieh. Probabilistic temporal logic for motion planning with resource threshold constraints. In Proc. of RSS, 2012.

[31] C. Yoo, R. Fitch, and S. Sukkarieh. Online task planning and control for aerial robots with fuel constraints in winds. In Proc. of WAFR, 2014. 